University of Nebraska - Lincoln

DigitalCommons@University of Nebraska - Lincoln

\title{
$5-2010$
}

\section{Substrate dependent buffer-layer assisted growth of nanoclusters}

Jan Honolka

Max-Planck Institute for Solid State Research, honolka@fzu.cz

Violetta Sessi

Max-Planck Institute for Solid State Research, violetta.sessi@jku.at

Jian Zhang

Max-Planck Institute for Solid State Research

Simon Hertenberger

Max-Planck Institute for Solid State Research, simon.hertenberger@wsi.tum.de

Axel Enders

University of Nebraska-Lincoln, a.enders@me.com

See next page for additional authors

Follow this and additional works at: https://digitalcommons.unl.edu/physicsenders

Part of the Physics Commons

Honolka, Jan; Sessi, Violetta; Zhang, Jian; Hertenberger, Simon; Enders, Axel; and Kern, Klaus, "Substrate dependent buffer-layer assisted growth of nanoclusters" (2010). Axel Enders Publications. 34.

https://digitalcommons.unl.edu/physicsenders/34

This Article is brought to you for free and open access by the Research Papers in Physics and Astronomy at DigitalCommons@University of Nebraska - Lincoln. It has been accepted for inclusion in Axel Enders Publications by an authorized administrator of DigitalCommons@University of Nebraska - Lincoln. 


\section{Authors}

Jan Honolka, Violetta Sessi, Jian Zhang, Simon Hertenberger, Axel Enders, and Klaus Kern 


\title{
Substrate dependent buffer-layer assisted growth of nanoclusters
}

\author{
Jan Honolka, ${ }^{1}$ Violetta Sessi, ${ }^{1}$ Jian Zhang, ${ }^{1,3}$ Simon Hertenberger, ${ }^{1}$ Axel Enders, ${ }^{2}$ and Klaus Kern ${ }^{1}$ \\ 1. Max-Planck Institute for Solid State Research, Heisenbergstrasse 1, 70569 Stuttgart, Germany \\ 2. Department of Physics and Astronomy and Nebraska Center for Materials and Nanoscience, \\ University of Nebraska-Lincoln, Lincoln, Nebraska 68588, USA \\ 3. School of Material Science and Engineering, Hebei University of Technology, 300130 TianJin, P.R. China
}

Corresponding author — J. Honolka, email j.honolka@fkf.mpg.de, tel 49711689 1617, fax 497116891662

\begin{abstract}
The role of the substrate on the morphology of nanometer size clusters fabricated by buffer layer assisted growth (BLAG) was studied using scanning tunneling microscopy. Clusters of Fe and Co were deposited on $\mathrm{Ag}(111), \mathrm{Cu}(100), \mathrm{Rh}(111)$, and $\mathrm{Pt}(111)$ surfaces using identical BLAG parameters, which are temperature, as well as metal and buffer layer coverage. Semi-hemispherical clusters are found on $\mathrm{Ag}(111)$ and $\mathrm{Cu}(100)$, while flat monolayer high islands are observed on $\mathrm{Rh}(111) \mathrm{and} \mathrm{Pt}(111) \mathrm{due}$ to complete wetting. The results of this study are in agreement with the common notion that BLAG is useful to deposit clusters of virtually any material on any substrate, however, the shape, size, and lateral distribution of the resulting clusters depend strongly on the substrate surface free energy and diffusion processes.
\end{abstract}

\section{Introduction}

Metal clusters and small nanoparticles are currently in the focus of extensive research, as they can exhibit physical or chemical properties that are distinctively different from the properties of the bulk and in the limit of single atoms. This offers opportunities for fundamental research of the dependence of electronic, magnetic, catalytic, and other properties on size and local coordination, and has great potential for many applications. Clusters in contact with surfaces have properties that are not only dependent on their size alone, but also on the epitaxial orientation, lattice mismatch, bond formation, or electronic hybridization which all can, in principle, affect the properties of the cluster or the substrate [1]. The two commonly used techniques to deposit clusters onto a substrate under vacuum conditions are the soft-landing of clusters formed in the gas phase [2], as well as noble gas buffer layer assisted cluster growth (BLAG) [3-5]. Both techniques are assumed to be suitable to form clusters of virtually any material on any substrate [6,7], since the clusters are formed in both methods before making contact with the substrate.

The BLAG has the advantage over the deposition of clusters from the gas phase that no experimental equipment other than standard molecular beam epitaxy (MBE) is required. BLAG-prepared clusters have been studied extensively in the past, as a function of buffer layer thickness or cluster material. Another advantage of BLAG is its ability to "grow anything on anything" [6, 7], which was demonstrated on weakly interactive substrates with low surface free energies, e.g., carbon-based [8], Si(111) [9], or $\mathrm{Au}(111)$ [10] surfaces. Here, diffusion effects during physical vapor deposition of metals on the Xe were mainly discussed. It has been reported that isolated 3D clusters are obtained on the substrate with a cluster size distribution that depends mainly on the used amount of cluster material and on the thickness of the noble gas buffer layer.

In this work we study the cluster size and morphology after BLAG on different substrates using identical amounts of evaporated metal and noble gas coverages. We will show that the choice of the substrate is critical for the size and morphology of the clusters in contact with the substrates. Our key conclusions are that:

(a) The final cluster shape is governed by the surface free energy of the substrate. While for low surface energies semi-hemispherical clusters comparable to those reported in the literature are observed, partial or complete wetting is observed for substrates with higher surface free energy, resulting in flat, 2D islands.

(b) Clusters are randomly distributed over the surface if the surface free energy is high. For lower surface free ener- 
gies, clusters are found to arrange in groups or even tend to decorate the step edges of the substrate, suggesting some cluster mobility and diffusion.

(c) Clusters can be frozen in non-equilibrium states at temperatures as high as room temperature.

\section{Experimental}

The $3 \mathrm{~d}, 4 \mathrm{~d}$ and $5 \mathrm{~d}$ metal substrates $\mathrm{Cu}(100), \operatorname{Ag}(111)$, $\mathrm{Rh}(111)$, and $\mathrm{Pt}(111)$, are prepared using standard sputtering and annealing recipes and the cleanliness was checked using Auger electron spectroscopy (AES) and scanning tunneling microscopy (STM).

For the fabrication of $3 \mathrm{~d}$ metal clusters we followed a procedure that is described in detail elsewhere [11]. In short, Fe or Co deposited on the substrate form small clusters on a substrate that has been pre-covered with a Xenon buffer gas layer at 35-40 K. Thermal desorption of the Xe at $T>100 \mathrm{~K}$ brings the clusters into contact with the substrate. The thickness of the Xe buffer layer is controlled by adjusting the Xe partial pressure and the exposure time. It has been demonstrated that a Xe gas flow of 5.5 Langmuir $\left(1 \mathrm{~L}=10^{-6}\right.$ Torr $\left.\times 1 \mathrm{~s}\right)$ leads to the formation of $1 \mathrm{ML}$ $\mathrm{Xe}$, assuming a sticking coefficient of 1 at the deposition temperature of $35 \mathrm{~K}$ [5]. However, the sticking coefficient might become smaller with increasing Xe thickness especially due to a decreasing Xe-substrate interaction. Reliable measurements of the Xe coverage as a function of Xe exposure for large coverage are not available. We therefore quote the Xe coverage, $\mu_{\mathrm{Xe}^{\prime}}$ in units of Langmuir (L) throughout this work.

Fe and Co was evaporated from a rod with a purity of 99.99\% using an electron beam evaporator. The deposition rates of the evaporators were calibrated by a quartz microbalance prior to each deposition. A nominal Fe coverage of $\mu_{\mathrm{Fe}}=1 \mathrm{ML}$ denominates an atomic density of $1.21 \times 10^{15}$ atoms $/ \mathrm{cm}^{2}$, which corresponds to the atomic density of a relaxed Fe bcc layer of $1.43 \AA$ thickness. For Co, the relation $1 \mathrm{ML}=1.6 \AA$ was used. Imaging of the substrates and clusers was done with a variable temperature STM. Data evaluation was partially done with WSxM software [12].

5,5'-bis(4-pyridyl)(2,2'-bipyrimidine) (PBP) molecules were evaporated from a crucible by resistive heating, while the substrate was held at room temperature. The evaporation temperature for PBP was about $500 \mathrm{~K}$.

\section{Results}

\subsection{Buffer-layer assisted growth on pristine metal surfaces}

The goal of the STM study in this section is to identify the dependence of the cluster morphology and distribution on the substrate material. To this end, Fe and Co clusters have been prepared on $\mathrm{Ag}(111), \mathrm{Cu}(100), \mathrm{Rh}(111)$, and $\mathrm{Pt}(111)$ substrates using 0.1 ML of Fe or Co and $10 \mathrm{~L}$ of Xe as buffer layer. All STM images shown were taken at room temperature and extracted cluster height distributions are summarized in Table 1.
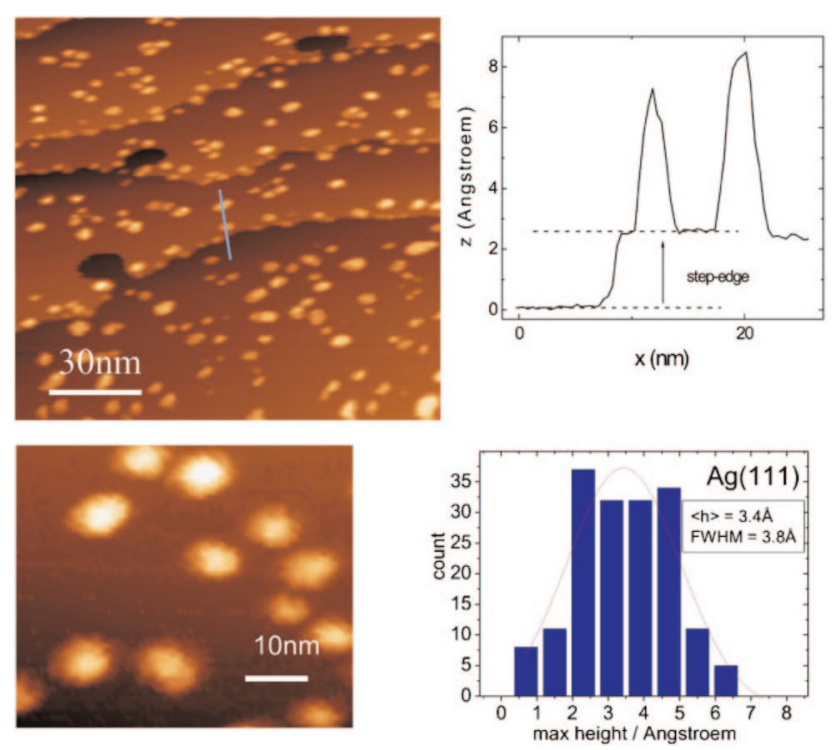

Figure 1. (BLAG of $0.1 \mathrm{ML} \mathrm{Fe} / 10 \mathrm{~L} \mathrm{Xe/Ag(111).} \mathrm{The} \mathrm{lateral} \mathrm{clus-}$ ter shape is round and the average height corresponds to about $2 \mathrm{ML}$. A template effect for smaller clusters is evident at the step-edges.

Table 1. Summary of cluster sizes (from STM) and lattice parameters.

\begin{tabular}{lrrrr}
\hline sample & $\begin{array}{r}\text { cluster height } \\
\langle h\rangle(\AA)\end{array}$ & $\begin{array}{r}\text { FWHM } \\
(\AA)\end{array}$ & $\begin{array}{r}\text { fcc lattice } \\
\text { mismatch }\end{array}$ & $\begin{array}{r}\text { surface NN } \\
\text { distance }(\AA)\end{array}$ \\
\hline $\mathrm{Fe} / \mathrm{Ag}(111)$ & 3.4 & 3.8 & $13.6 \%$ & 2.88 \\
$\mathrm{Fe} / \mathrm{Cu}(100)$ & 1.6 & 1.4 & - & 2.55 \\
$\mathrm{Fe} / \mathrm{Rh}(111)$ & 0.9 & 0.4 & $7.5 \%$ & 2.69 \\
$\mathrm{Co} / \mathrm{Pt}(111)$ & 2.3 & 0.6 & $9.4 \%$ & 2.77 \\
\hline
\end{tabular}

Summarized in Figure 1 are the results for Fe clusters on $\operatorname{Ag}(111)$. The clusters exhibit a 3D semi-hemispherical shape, as can be seen from the line profile. Statistical data on the cluster height, i.e., the maximum $z$-height above the substrate terrace as obtained from several STM images, are also given in Figure 1. The histogram shows that the average height $\langle h\rangle$ of the clusters is about $3.4 \AA$ corresponding to $\sim 2 \mathrm{ML}$ of Fe. The full width half maximum (FWHM) of the height distribution is $3.8 \AA$. Moreover, the clusters seem to preferentially populate the top of the surface steps. The presence of mainly smaller clusters along the step-edges proves the presence of a considerable cluster mobility during or after the BLAG process.

Similar results are observed for BLAG on $\mathrm{Cu}(100)$, as can be seen in Figure 2. Also here, compact clusters are observed along with a decoration of the step-edges. The height histogram shows an average cluster height only $\sim 2.3 \AA$, which is lower than in the case of $\mathrm{Ag}(111)$ and corresponds to about 1-2 ML Fe. Also, the average cluster diameter is clearly reduced leading to an increased areal density on the surface. 

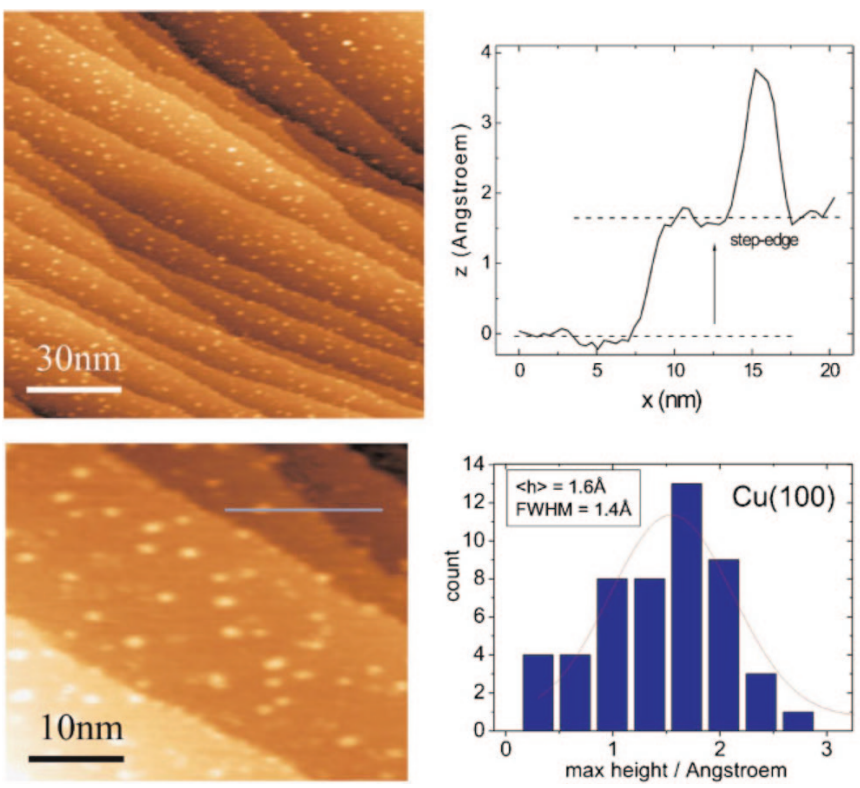

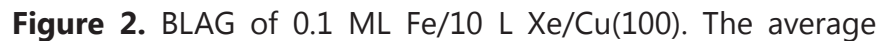
height corresponds to about $1 \mathrm{ML}$. Again a template effect for smaller clusters is visible at the step-edges.

A qualitatively different result is obtained for BLAG systems $\mathrm{Fe} / \mathrm{Rh}(111)$ and $\mathrm{Co} / \mathrm{Pt}(111)$, as shown in Figures 3 and 4 . The STM images show ramified island shapes especially in the case of $\mathrm{Rh}(111)$ versus round perimeters of the clusters as in Figures 1 and 2. Moreover, on $\mathrm{Rh}(111)$ no step-edge decoration is observed. Also in the case of $\mathrm{Pt}(111)$ the zoom-in in Figure 4 shows that the decoration effect is significantly reduced. Here, only very few smaller clusters have migrated to the step-edge. Besides the larger clusters some small impurity-like clusters of sub-nanometer size are visible on both $\mathrm{Rh}(111)$ and $\mathrm{Pt}(111)$. All these observations indicate that the mobility of both single $3 \mathrm{~d}$ atoms and clusters as a whole is significantly reduced or absent on $\mathrm{Rh}(111)$ and $\mathrm{Pt}(111)$. We observe an average height $\langle h\rangle=1.9 \AA$ for Co on Pt, which corresponds to clusters of monolayer height. On $\mathrm{Rh}(111)$, however, the $h$ amounts to only $0.9 \pm 0.4 \AA$, which is less than the expected height of one monolayer of $\mathrm{Fe}$. The absence of dislocation lines, despite the large lattice mismatch of $-7.5 \%$ for $\mathrm{Fe} / \mathrm{Rh}(111)$, is in agreement with observations published earlier [13].

We conclude that the reduced cluster height to below $1 \mathrm{ML}$ is the result of a relatively high electronic density of states of the Rh surface. An alternative explanation could be the intermixing alloy formation, where the alloy would appear with an apparent height different from that of a $\mathrm{Fe}$ monolayer. However, such alloy formation has not been reported at room temperature. Studies with angular resolved photoemission spectroscopy measurements (ARUPS) showed that Fe deposited at $300 \mathrm{~K}$ formed an alloy with $\mathrm{Rh}(111)$ only after annealing at $500 \mathrm{~K}$ [14].
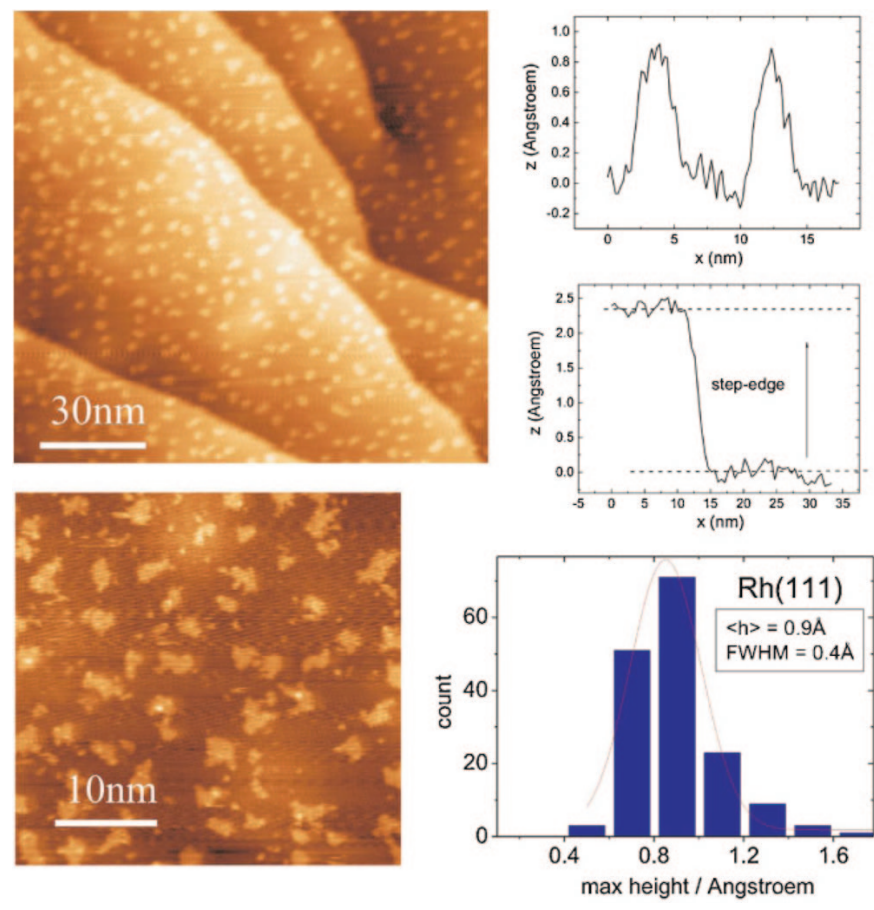

Figure 3. BLAG of $0.1 \mathrm{ML} \mathrm{Fe/10} \mathrm{L} \mathrm{Xe/Rh(111).} \mathrm{No} \mathrm{template}$ effect is visible and the clusters grow in 2D with a ramified lateral shape.
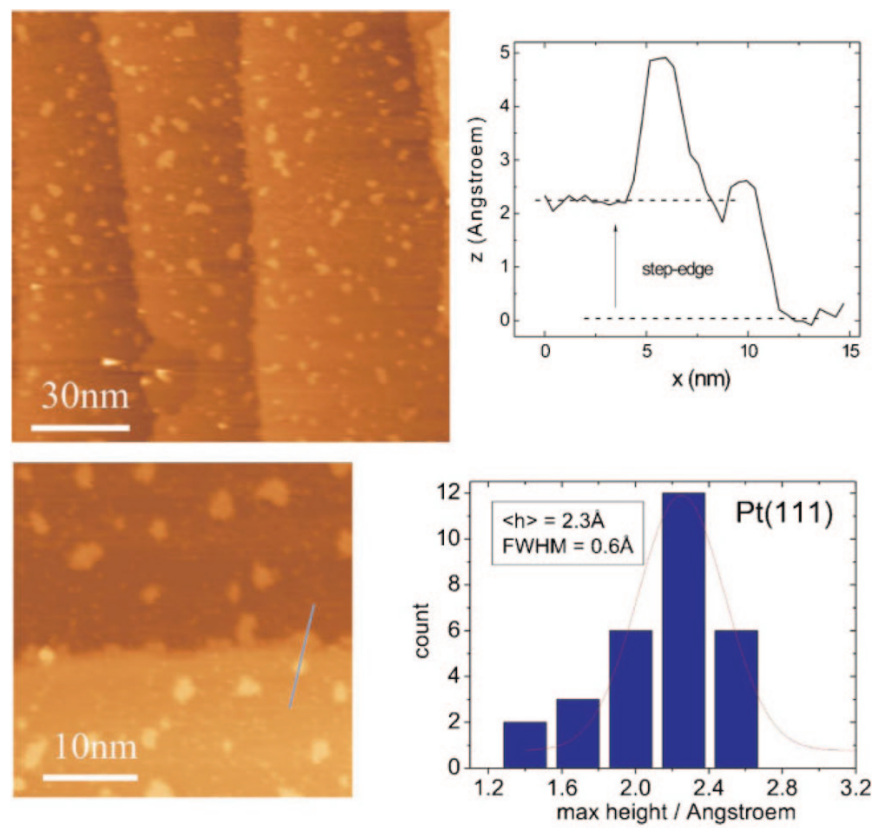

Figure 4. $B L A G$ of $0.1 \mathrm{MLCO} / 10 \mathrm{~L} \mathrm{Xe/Pt(111).} \mathrm{No} \mathrm{template}$ effect is visible and the clusters grow in 2D with a ramified lateral shape.

The final cluster shape observed with STM at room temperature is discussed on the basis of the surface free energies of the different substrates. Using the Young-Dupré YD) formula for wetting processes at surfaces, the contact angle of a droplet can be estimated. This relation is often used for the growth of thin films at or near thermal equi- 
librium, but should also give a reasonable estimate for the wetting behavior of the $3 \mathrm{~d}$ cluster. According to $\mathrm{YD}$, the interfacial energy $\gamma_{\mathrm{AB}}^{0}$ between two materials $\mathrm{A}$ and $\mathrm{B}$ can be expressed as:

$$
r_{\mathrm{AB}}^{0}=\gamma_{\mathrm{A}}^{0}+\gamma_{\mathrm{B}}^{0}+\Delta \gamma_{\mathrm{AB}}^{\mathrm{ads}}
$$

where $\gamma_{i}^{0}$ is the surface free energy of material $i$ and $\Delta \gamma_{\mathrm{AB}}^{\mathrm{ads}}$ the interfacial adhesion of the system AB. From Reference 15 the interfacial adhesion can be expressed as $\Delta \gamma_{A B}^{\text {ads }}$ $=-2 \varphi\left(\gamma_{\mathrm{A}}^{0} \times \gamma_{\mathrm{B}}^{0}\right)^{1 / 2}$ with $\varphi$ being a dimensionless, material dependent parameter of about unity in the case of the similar systems studied in this work. With Equation (1) we can thus calculate the values $\gamma_{\mathrm{AB}}^{0}$ for the respective systems, using the surface free energies $\gamma_{i}^{0}$ given in Reference 15 and obtain $\gamma_{\mathrm{AB}}^{0}=1.53,0.89,-0.18$, and $0.007 \mathrm{~J} / \mathrm{m}^{2}$ for $\mathrm{Fe} /$ $\mathrm{Ag}, \mathrm{Fe} / \mathrm{Cu}, \mathrm{Fe} / \mathrm{Rh}$, and $\mathrm{Co} / \mathrm{Pt}$, respectively. The very small interfacial energy for $\mathrm{Co} / \mathrm{Pt}$ interfaces indicates a tendency towards layer-by-layer growth, or here, for preferred wetting of the substrate with the cluster material. Calculations of the relevant energies also allowed us to estimate the equilibrium morphology of the system using the YD formula for a liquid (L) droplet on a solid (S) as a function of the surface free energies of the $S$ and $L$ and the interfacial energy of the S-L system:

$$
r_{\mathrm{S}}^{0}=r_{\mathrm{LS}}^{0}+r_{\mathrm{L}}^{0} \cos \theta
$$

$\theta$ defines the contact angle of the droplet on the surface. Special cases are complete wetting for $\theta=0^{\circ}$, and a crossover from wetting to dewetting for $\theta=90^{\circ}$. In case that $r_{\mathrm{S}}^{0}>\gamma_{\mathrm{LS}}^{0}+\gamma_{\mathrm{L}}^{0}$ Equation (2) is not valid and another parameter $\hat{S}$ has to be defined [16]:

$$
\hat{S}=r_{\mathrm{S}}^{0}-\gamma_{\mathrm{LS}}^{0}-\gamma_{\mathrm{L}}^{0}
$$

For $\hat{S} \geq 0$ complete wetting occurs, while for $\hat{S} \ll 0$ droplets will form. We estimate a perfect wetting for $\mathrm{Fe} / \mathrm{Rh}$ $(\hat{S}>0)$ and $\mathrm{Co} / \mathrm{Pt}\left(\theta=0^{\circ}\right.$, adhesion), and partial wetting in the case of $\mathrm{Fe} / \mathrm{Ag}\left(\theta=66.0^{\circ}\right)$ and $\mathrm{Fe} / \mathrm{Cu}\left(\theta=53.3^{\circ}\right)$ in fair agreement with our STM observations. The surface free energy related parameters discussed above, as well as the calculated contact angles, are summarized in Table 2.

The problem of diffusion of clusters is closely related to the well-known problem of the diffusion of single atoms at surfaces and will be discussed in the following. Several mechanisms for cluster diffusion have been described in the literature so far. In the case of 2D clusters, such as those observed on $\mathrm{Rh}(111)$ and $\mathrm{Pt}(111)$, the diffusion can be based on either single atom processes or collective movements.

Table 2. Surface free energy related parameters of the sample systems.

\begin{tabular}{lrrrr}
\hline sample & $\Delta \gamma_{A B}^{\text {ads }}\left(\mathrm{J} / \mathrm{m}^{2}\right)$ & $\gamma_{\mathrm{AB}}^{0}\left(\mathrm{~J} / \mathrm{m}^{2}\right)$ & $\hat{S}\left(\mathrm{~J} / \mathrm{m}^{2}\right)$ & $\Theta\left(^{\circ}\right)$ \\
\hline $\mathrm{Fe} / \mathrm{Ag}(111)$ & -3.57 & 1.53 & - & 66.0 \\
$\mathrm{Fe} / \mathrm{Cu}(100)$ & -4.21 & 0.89 & - & 53.3 \\
$\mathrm{Fe} / \mathrm{Rh}(111)$ & -5.28 & -0.18 & 0.18 & - \\
$\mathrm{Co} / \mathrm{Pt}(111)$ & -5.30 & 0.007 & - & 0 \\
\hline
\end{tabular}

In the former case the known mechanisms are sequential displacements, edge diffusion, or simultaneous evaporation and condensation [17]. The ramified lateral shape of the clusters found on $\mathrm{Rh}(111)$ and $\mathrm{Pt}(111)$ indicates a low mobility of $3 \mathrm{~d}$ atoms along the cluster edge and negligible probability of evaporation of atoms from the clusters at room temperature. Also sequential displacements would allow the cluster to assume a compact lateral shape, which is not observed. Single atom processes thus seem to be significantly suppressed on $\operatorname{Rh}(111)$ and $\mathrm{Pt}(111)$, resulting in significantly reduced cluster mobility. We believe that despite the low mobility well-separated larger clusters are observed on these substrates because they form partly on the Xe layer in agreement with other works on BLAG in the literature. However, in the case of $\mathrm{Pt}(111)$ we have shown that for thin Xenon films Co entities of about a few atom size are already in contact with the metal substrate before the Xenon film is entirely desorbed [18]. This proves that in the case of strongly interacting substrates like $\mathrm{Pt}(111)$, entities tend to sink through the Xenon layer at an earlier stage. After warming the sample to room temperature these entities further aggregate to a certain extend via ripening processes [18].

On $\mathrm{Rh}(111)$ we do not have low temperature STM data. However, due to the fact that from all substrates studied in this work, $\mathrm{Rh}(111)$ shows the lowest single atom and cluster mobility, we attribute the presence of well-separated clusters to cluster aggregation at an early stage on or within the Xenon buffer layer.

The same BLAG parameters on $\operatorname{Ag}(111)$ and $\mathrm{Cu}(100)$ lead to a distinctively different morphology in that clusters remain in a semi-hemispherical shape, and preferentially decorate the step edges of the substrate. Both is indicative of atomic diffusion at the cluster edges, or cluster diffusion as a whole, for instance by evaporation-condensation processes.

It is concluded that the final shape of the clusters after making contact with the substrate during BLAG is dependent on surface wetting and de-wetting, which in turn depends on the surface free energies of the substrate and cluster material. Fe clusters on $\mathrm{Ag}(111)$ and $\mathrm{Cu}(100)$ substrates are compact, with semi-hemispherical shape, and mobile enough to decorate the steps. Clusters prepared using the same BLAG parameters on $\mathrm{Rh}(111)$ and $\mathrm{Pt}(111)$ completely wet the surface and are frozen in an irregular shape, suggesting the absence of certain diffusion mechanisms such as corner crossing. Cluster migration to the step edges can happen already on the Xe layer, but is more likely a result of cluster diffusion on the metal surface as the results from the next section will show.

\subsection{Buffer-layer assisted growth on molecular template layers}

A key question to answer is: What is the size and shape of the clusters while still situated on the Xe buffer, and how do these parameters change upon making contact with the metal substrate? Our conclusion from the previous section is that the clusters' shape and size do depend on wetting effects as well as on diffusion processes and ripening, 

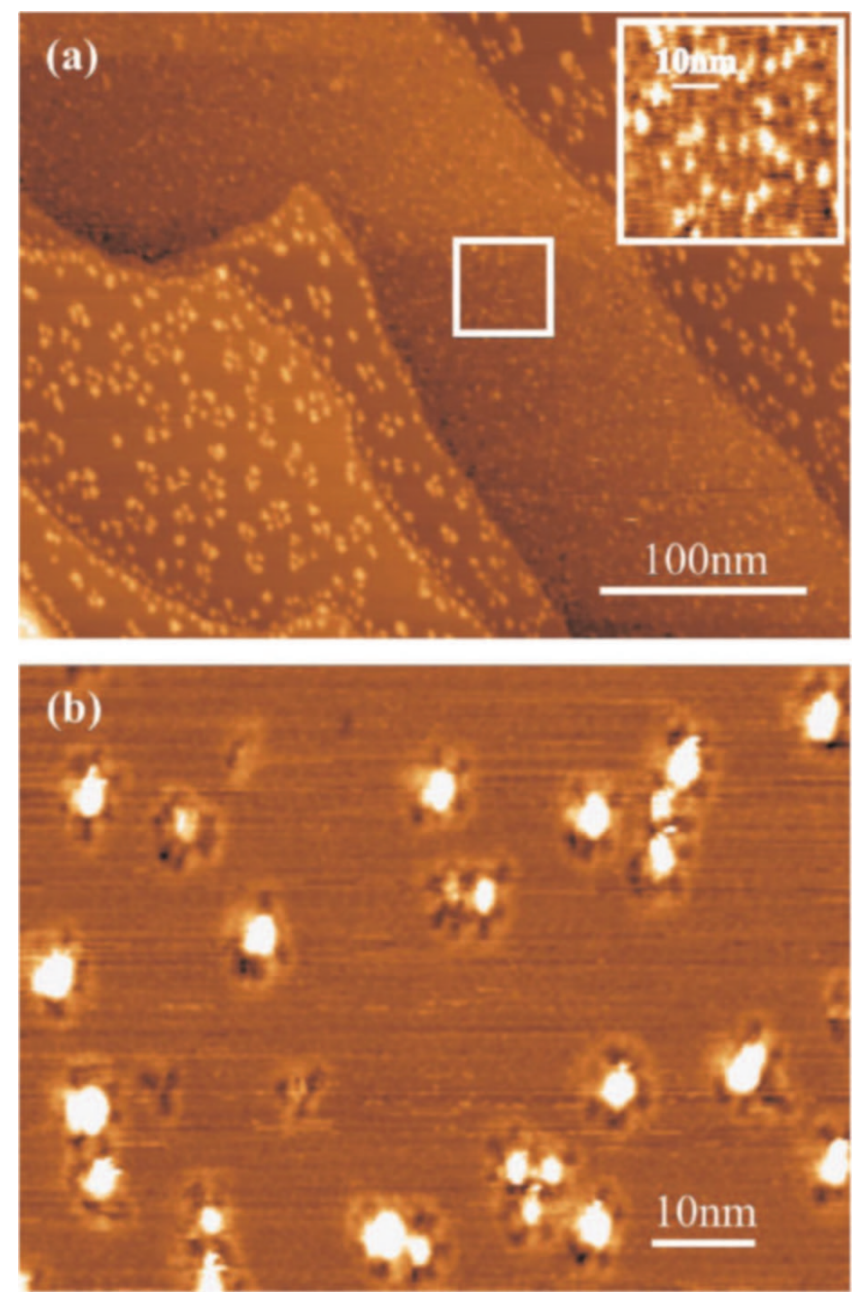

Figure 5. BLAG of $0.3 \mathrm{MLFe}+10 \mathrm{~L}$ Xe on $\mathrm{Ag}(111)$ which was pre-covered with PBP molecules. (a) On a large scale the separation into two phases is visible one being clusters on the bare $\mathrm{Ag}(111)$ substrate and the other clusters embedded in a dense molecule network. Inset: Zoom-in into the PBP covered area. (b) Zoom-in at the bare $\mathrm{Ag}(111)$ substrate.

which is mostly determined by the substrate. Our approach is now to preserve the clusters by depositing on a molecular template layer to suppress any diffusion processes after making contact with the substrate. To this end, the $\operatorname{Ag}(111)$ has been pre-covered with a sub-monolayer of PBP molecules prior to BLAG of clusters. PBP molecule have been found to self-organize into dense row structures on metal substrates at RT, even for sub-monolayer coverages. Adjacent rows of molecules are connected by intermolecular electrostatic or hydrogen bonding-like interaction of the type $\mathrm{CH} \cdots \mathrm{N}$ [19].

The STM image in Figure 5a shows clusters formed of $0.3 \mathrm{ML}$ of Fe on 0.5 ML PBP/Ag(111) using $10 \mathrm{~L}$ Xenon. Two phases can be clearly distinguished. One corresponds to the known observation of compact clusters on pristine $\operatorname{Ag}(111)$ similar to those in Figure 1, showing the known features step decoration and formation of cluster groups. A zoom into this area, shown in Figure 5b, reveals that these clusters are decorated by single molecules. The second phase consists of considerably smaller clusters, embedded in the layer of molecules, see inset.

We interpret the observed smaller cluster size on the molecular network as in the inset of Figure 5 in terms of inhibited diffusion. When the clusters make contact with the molecular layer during Xe desorption at approximately $100 \mathrm{~K}$, any ripening or diffusion is most likely suppressed when further increasing the temperature to room temperature. We thus argue that on the PBP network the clusters are preserved in their original size as on the Xe layer since thermally activated diffusion or ripening is inhibited. Comparing the size and areal density of clusters in both phases, it is obvious that the clusters on the molecule layer are randomly distributed, significantly smaller, and do not form cluster islands or decorate the steps. An estimate of the cluster height and width is complicated by the fact that clusters might be partially buried in the molecule layer, and because the molecular layer is not a good reference surface for height measurements due to its roughness and low electronic density. At intermediate temperatures, single molecules are evaporated from the molecular network and condense at the larger Fe clusters formed on the bare $\mathrm{Ag}(111)$ substrate, as seen in Figure 5b.

The important conclusion of the result of the moleculeassisted experiments is the observation of significantly reduced cluster sizes on the molecular phase due to suppression of ripening processes. However, we want to comment on possible differences in the desorption dynamics of Xenon on the molecular layer and the metal substrate. As shown by Palmer et al. [20] significant diffusion, restructuring, and dewetting of the Xe occur prior to desorption which can be substrate dependent. We expect van-der-Waals forces on the bare $\operatorname{Ag}(111)$ substrate to be larger compared to the molecular layer. The Xenon layer in direct contact with the underlying molecular network will thus desorb at slightly lower temperature as compared to the bare substrate. This can influence the cluster size to a certain extent as shown in Reference 19.

\subsection{Comparison of clusters formed by BLAG with epi- taxially grown clusters}

Evidently, the size and shape of the clusters obtained by BLAG is dependent on wetting and ripening after making contact with the substrate surface. Yet, clusters formed by BLAG are distinctively different from epitaxially grown islands, as will be shown in this section. We compare the growth of BLAG-grown Co clusters on Pt(111) with epitaxially grown Co islands on the same substrate. Two typical STM images for BLAG clusters and epitaxially grown Co are summarized in Figure 6. The epitaxial growth for the sample in Figure $6 \mathrm{~b}$ was done with the substrate held at $130 \mathrm{~K}$, which corresponds roughly to the temperature where $\mathrm{Xe}$ is completely desorbed during BLAG. In both cases the cluster height is about $2.3 \AA$, corresponding to a complete wetting of the substrate and the formation of monolayer islands. Differences, however, are visible again in the lateral shape. Direct deposition leads to well-known triangular shaped Co monolayer islands (Figure 6b with a 
a)
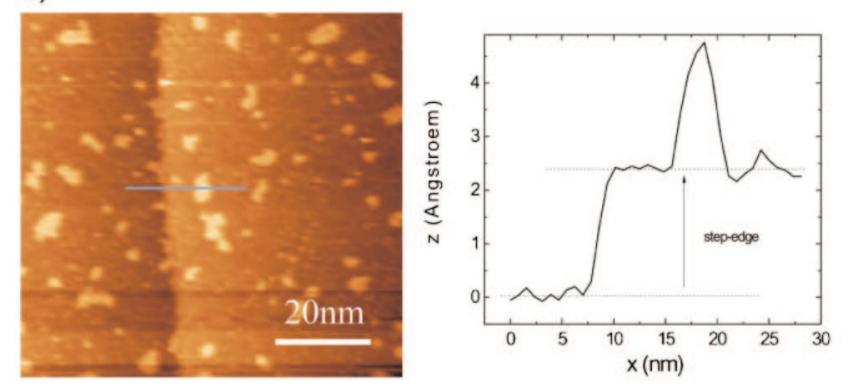

b)

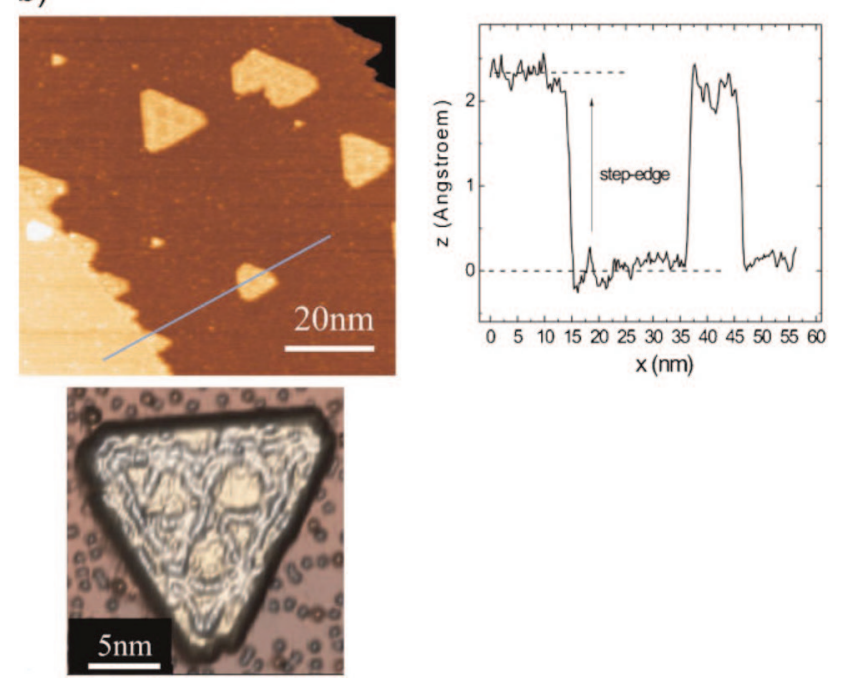

Figure 6. Comparison of the island shape for BLAG grown clusters (a), and direct deposition of Co atoms on $\mathrm{Pt}(111)$ at substrate temperatures of $130 \mathrm{~K}$ (b). All STM images were taken at $300 \mathrm{~K}$. The enlarged island in the lower right corner shows characteristic reconstruction patterns due to the lattice mismatch between $\mathrm{Co}$ and Pt.

characteristic reconstruction pattern induced by the lattice mismatch of $9.4 \%$ between Co and the Pt surface). For further details on the reconstruction pattern we refer to Reference 21. In comparison, the BLAG clusters are significantly smaller and of irregular shape. Here, no reconstruction pattern is observed. We conclude that the BLAG clusters were not able to assume the energetically preferred triangular shape, most likely due to suppressed edge diffusion along the island edges during and after the BLAG process. In other words, the non-equilibrium shape, which is a result from cluster deposition at low temperatures, remains partly frozen in during warming up the sample to RT.

\section{Summary}

In this paper, the BLAG of sub-monolayer amounts of $3 \mathrm{~d}$ metals on four different metal substrate surfaces was investigated. We find significant differences in the cluster morphology between the substrates studied, which we discuss as the result of differences in the surface free energies and its consequences for diffusion processes on the surfaces. We succeeded in suppressing such diffusion of clusters by performing BLAG on a molecular network, thus preserving their original size as formed on the $\mathrm{Xe}$ buffer layer.

Further, a comparison of BLAG clusters with MBEgrown $\mathrm{Co}$ islands shows that nucleation and growth during MBE results in large islands of regular shape, as a result of edge diffusion. In contrast, such processes are suppressed if the clusters are deposited as a whole, thus freezing in a non-equilibrium state of the clusters which remains up to room temperature. Our results are in agreement with the notion that BLAG is a useful method to deposit clusters of virtually any material on any substrate, but we find significant differences in the final cluster size and shape between the substrates studied.

Acknowledgments - We thank the Deutsche Forschungsgemeinschaft (DFG) for funding within the Schwerpunktprogramm "Clusters in contact with surfaces," SPP 1153. This work was further supported by NSF CAREER (DMR0747704) and NSF MRSEC (DMR-0213808).

\section{References}

[1] J. P. Pierce, M. A. Torija, Z. Gai, J. Shi, T. C. Schulthess, G. A. Farnan, J. F. Wendelken, E. W. Plummer, and J. Shen, Phys. Rev. Lett. 92, 237201 (2004).

[2] H. Brune, W. Harbich, R. Monot, J. Buttet, K. Kern, K. Bromann, and C. Felix, Science 274, 956 (1996).

[3] J. H. Weaver and G. D. Waddill, Science 251, 1444 (1991).

[4] J. H. Weaver, L. Huang, and S. J. Chey, Phys. Rev. Lett. 80, 4095 (1998).

[5] J. H. Weaver and C. Haley, Surf. Sci. 518, 243 (2002).

[6] J. H. Weaver and V. N. Antonov, Surf. Sci. 557, 1 (2004).

[7] G. Kerner and M. Asscher, Surf. Sci. 557, 5 (2004).

[8] V. N. Antonov, Phys. Rev. B 68, 205418 (2003).

[9] L. Huang, S. Jay Chey, and J. H. Weaver, Phys. Rev. Lett. 80, 4095 (1998).

[10] M. Asscher, Surf. Sci. 603, 957 (2009).

[11] J. Zhang et al., Eur. Phys. J. D 45, 515 (2007).

[12] I. Horcas, R. Fernández, J. M. Gómez-Rodríguez, and J. Colchero, Rev. Sci. Instrum. 78, 013705 (2007).

[13] Anne Lehnert, "Magnetism of Individual Adatoms and of Epitaxial Monolayers", THÈSE NO 4411(2009) Ecole Polytechnique Federale de Lausanne (EPFL).

[14] K. Homann, H. Kuhlenbeck, and H.-J. Freund, Z. Phys. Chem. 198, 135 (1997).

[15] A. R. Miedema and B. E. Nieuwenhuys, Surf. Sci. 104, 491 (1981).

[16] A. R. Miedema and B. E. Nieuwenhuys, Surf. Sci. 251, 635 (1991).

[17] K. Oura, V. G. Lifshits, A. A. Saranin, A. V. Zotov, and M. Katayama, in: Surface Science - An introduction, Advanced Texts in Physics (Springer-Verlag, Berlin, Heidelberg, 2003), chap. 13.

[18] V. Sessi, in press (2009), cond-mat/arXiv:0908.4365.

[19] S. L. Tait, A. Langner, N. Lin, R. Chandrasekar, M. Ruben, and K. Kern, Chem. Phys. Chem. 9, 2495 (2008).

[20] J. S. Palmer, P. Swaminathan, S. Babar, and J. H. Weaver, Phys. Rev. B 77, 195422 (2008).

[21] F. Meier et al., Phys. Rev. B 74, 195411 (2006). 